\title{
Long-chain $n$-3 PUFA in vegetarian women: a metabolic perspective
}

\author{
Graham C. Burdge ${ }^{1}$, Sze-Yen $\operatorname{Tan}^{2}$ and Christiani Jeyakumar Henry ${ }^{2}$ \\ ${ }^{1}$ Academic Unit of Human Development and Health, Faculty of Medicine, University of Southampton, Southampton SO16 6YD, UK \\ ${ }^{2}$ Clinical Nutrition Research Centre, Centre for Translational Medicine, Yong Loo Lin School of Medicine, Singapore and Department of \\ Biochemistry, National University of Singapore, Singapore
}

(Received 5 June 2017 - Final revision received 14 September 2017 - Accepted 9 October 2017)

Journal of Nutritional Science (2017), vol. 6, e58, page 1 of 8

doi:10.1017/jns.2017.62

Abstract

Vegetarian diets have been associated with health benefits, but paradoxically are low in EPA and DHA which are important for development, particularly of the central nervous system, and for health. Humans have limited capacity for synthesis of EPA and DHA from $\alpha$-linolenic acid, although this is greater in women than men. Oily fish and, to a lesser extent, dairy foods and meat are the primary sources of EPA and DHA in the diet. Exclusion of these foods from the diet by vegetarians is associated consistently with lower EPA and DHA status in vegetarian women compared with omnivores. The purpose of the present review was to assess the impact of low EPA and DHA status in vegetarian pregnancies on the development and health of children. EPA and DHA status was lower in breast milk and in infants of vegetarian mothers than those born to omnivore mothers, which suggests that in the absence of pre-formed dietary EPA and DHA, synthesis from $\alpha$-linolenic acid is an important process in determining maternal EPA and DHA status in pregnancy. However, there have been no studies that have investigated the effect of low maternal DHA status in vegetarians on cognitive function in children. It is important to address this gap in knowledge in order to be confident that vegetarian and vegan diets during pregnancy are safe in the context of child development.

Key words: EPA: DHA: Vegetarian women: $\boldsymbol{\alpha}$-Linolenic acid: Desaturase

\section{Introduction}

Vegetarianism is a widely practised dietary choice that may be adopted for cultural or religious reasons, or out of personal preference, possibly because of perceived health benefits or concerns about animal welfare ${ }^{(1)}$. The practice of vegetarianism encompasses a range of dietary choices such as the complete exclusion of all meat, fish and dairy produce (veganism), exclusion of meat and fish with inclusion of dairy products and eggs (ovo-lacto-vegetarianism), or exclusion of meat, fish and eggs, but with inclusion of dairy products (lactovegetarianism $)^{(1)}$. For the purpose of the present review, ovo-lacto-vegetarianism will be described as vegetarianism as this is the most common type of vegetarian diet that has been studied previously. There are too few studies that have investigated the effects of other vegetarian dietary practices on $n$-3 PUFA status, with the exception of veganism of which there have been a number of studies and will also be discussed here.

Exclusion of major food groups from the diet may confer a risk of low status or deficiency of nutrients that are found predominately or exclusively in the excluded foods. The $n-3$ PUFA EPA and DHA are obtained primarily from oily fish and, to a lesser extent, from meat and dairy products ${ }^{(2)}$. Thus excluding these foods from the diet may incur risk of low EPA and DHA status. Lower EPA and DHA intake and status have been associated with adverse effects on cardiovascular health and on inflammatory disease ${ }^{(3-5)}$. Furthermore, adequate assimilation of DHA by the developing central

Abbreviations: ALNA, $\alpha$-linolenic acid; FADS, fatty acid desaturase.

* Corresponding author: Professor G. C. Burdge, email g.c.burdge@soton.ac.uk

(C) The Author(s) 2017. This is an Open Access article, distributed under the terms of the Creative Commons Attribution licence (http://creativecommons.org/licenses/by/4.0/), which permits unrestricted reuse, distribution, and reproduction in any medium, provided the original work is properly cited. 
nervous system is required for optimal function ${ }^{(6)}$ and deficits in DHA accumulation have been associated with impaired retinal function in preterm infants ${ }^{(7)}$ and with altered behaviour in non-human primates ${ }^{(8-10)}$. There have been several previous reviews of the impact of vegetarian diets on EPA and DHA status, which concluded that individuals who follow vegetarian diets have lower EPA and DHA status compared with omnivores $^{(11-15)}$. Nevertheless, vegetarian diets have been associated with specific health benefits including reduction in CVD risk factors ${ }^{(16)}$, lower risk of type 2 diabetes mellitus and impaired glucose homeostasis ${ }^{(17,18)}$ and the metabolic syndrome $^{(19)}$, enhanced weight reduction, although effects on maintenance of lower weight are uncertain ${ }^{(20)}$, reduced risk of cancerr ${ }^{(21,22)}$, and differential effects of vegetarian and vegan diets on the gut microbiota ${ }^{(23)}$. Vegetarianism in affluent countries tends to be practised by individuals with higher income and educational attainment, while vegetarianism in less wealthy counties is associated primarily with poverty, and religious or cultural practice ${ }^{(24)}$. Thus, it has been suggested that at least part of the beneficial effects of vegetarianism on health may be attributed to lifestyle rather than dietary practice per se ${ }^{(24)}$.

DHA status has been shown to be higher in women than men ${ }^{(25)}$, partly because women have been shown to be able to convert the essential fatty acid $\alpha$-linolenic acid (ALNA) to $\mathrm{DHA}^{(26)}$, while such capacity in men is negligible ${ }^{(27)}$. It is thought that such metabolic capacity in women may facilitate the supply of DHA to their offspring. Because DHA is important for neurological development, low DHA status in non-pregnant and pregnant vegetarian women may adversely affect the development of children born to vegetarian mothers. The purpose of the present review was to compare the impact of vegetarian diets on EPA and DHA status in non-pregnant and pregnant women, and to assess whether maternal vegetarian diet affects the development of their children.

\section{n-3 Fatty acids: dietary sources}

n-3 Fatty acids are long-chain PUFA that are characterised by the presence of a double bond on the third carbon from the methyl end of the hydrocarbon chain. They can be grouped into the 18-carbon fatty acids ALNA and stearidonic acid (18:4n-3) that are present in vegetable oils, and those with chains of 20 or more carbons found in animal products, especially oily fish: $20: 4 n-3$, EPA, DPA $n-3(22: 5 n-3)$ and DHA. ALNA is synthesised in plants from oleic acid $(18: 1 n-9)$ by sequential reactions that are catalysed by $\Delta 12$ and $\Delta 15$ desaturases ${ }^{(28)}$. Plants of the Boraginaceae are able to synthesise stearidonic acid by $\Delta 6$ desaturase activity, and may contain between 5 and $10 \%$ of this PUFA $^{(29)}$. Humans and other mammals are unable to synthesise ALNA because they lack $\Delta 12$ and $\Delta 15$ desaturases and consequently this fatty acid is regarded as essential in the diet ${ }^{(30)}$. Poor ALNA intake, primarily in patients receiving total parenteral nutrition, has been linked to specific deficiency symptoms ${ }^{(31)}$. Therefore, in order to maintain adequate EPA and DHA status, humans and other mammals are dependent upon either consuming these fatty acids pre-formed in the diet from animal-derived foods ${ }^{(2)}$ and/or on synthesis from ALNA.

\section{Synthesis of EPA and DHA in humans}

The consensus pathway for conversion of ALNA to longer-chain $n$-3 PUFA in mammals was elucidated by Voss \& Sprecher ${ }^{(32)}$ in rat liver and involves sequential desaturation and carbon chain elongation reactions. The initial rate-limiting reaction introduces a double bond into ALNA at the $\Delta 6$ position and is catalysed by $\Delta 6$ desaturase to produce $18: 4 n-3$, which is then converted to $20: 4 n-3$ by the addition of two carbons by elongase 5 activity. Desaturation of $20: 4 n-3$ to form EPA is catalysed by $\Delta 5$ desaturase. Chain elongation of EPA to DPA $n-3$ is catalysed by elongase 2 or 5 , and then DPA $n-3$ can be elongated to $24: 5 n-3$ by elongase 2 activity. Desaturation of $24: 5 n-3$ at the $\Delta 6$ position by $\Delta 6$ desaturase produces $24: 6 n-6$. Then $24: 6 n-3$ is translocated from the endoplasmic reticulum to peroxisomes where two carbons are removed to form DHA. It has been suggested that the reactions downstream of DPA $n-3$ may regulate DHA synthesis independent of the synthesis of DPA $n-3^{(32)}$.

Although this pathway is active in rodents, and possibly other species, humans appear to have limited capacity for the synthesis of EPA, DPA $n-3$ and, in particular, DHA. Dietary supplementation studies in men alone or men plus postmenopausal women show an increment in EPA in blood lipids that was related to the level of ALNA intake, but no increase in $\mathrm{DHA}^{(33)}$. These findings are supported by the results of studies in which men who consumed ALNA labelled with a stable isotope tracer showed synthesis of EPA, DPA $n-3$, but not DHA ${ }^{(27)}$. In addition, James $e t$ al. ${ }^{(34)}$ conducted a study to attempt to overcome the point of constraint in ALNA conversion. Men and postmenopausal women consumed either ALNA, $18: 4 n-3$, which would be expected to overcome any constraint in desaturation of ALNA by $\Delta 6$ desaturase, or EPA, which would be expected to overcome any constraint in conversion due to $\Delta 6$ desaturase, $\Delta 5$ desaturase and elongase 5 . All three interventions induced an increase in EPA and DPA $n-3$ status, but not in $\mathrm{DHA}^{(34)}$. In some dietary supplementation studies, feeding ALNA, $18: 4 n-3$ or EPA was associated with a decrease in DHA status ${ }^{(33)}$. This may reflect retro-conversion of DHA to shorter-chain, less unsaturated $n-3$ PUFA in the absence of dietary input of pre-formed DHA ${ }^{(35)}$. Based on these findings, it has been concluded that capacity for conversion of ALNA to EPA is limited and to DHA is severely constrained in men and post-menopausal women ${ }^{(26)}$. However, conversion of stable isotope-labelled ALNA to EPA and DHA was markedly greater in young women than in men ${ }^{(36)}$. This apparent sex difference is supported by a meta-analysis of fifty-one studies which showed that typically women have a $20 \%$ higher proportion of DHA in blood lipids than men ${ }^{(25)}$. Higher DHA status in females compared with males has also been reported in rodents and wild birds (Parus major) ${ }^{(37)}$, which suggests that this sex difference may either be conserved or the result of convergent evolution. If so, this implies that higher DHA status and capacity for synthesis is of biological importance in females. Furthermore, female sex hormones, specifically $17-\alpha$-ethynyl oestradiol and progesterone have been shown to increase DHA synthesis ${ }^{(36)}$ or are associated with higher 
DHA status ${ }^{(38,39)}$. A recent study in HepG2 cells and human primary hepatocytes showed that progesterone, but not $17-\alpha$-ethynyl oestradiol or testosterone, increased EPA, DPA $n-3$ and DHA synthesis, up-regulated the mRNA expression of fatty acid desaturase 1 (FADS1) and FADS2, which encode $\Delta 5$ or $\Delta 6$ desaturases, respectively, and elongation of very long-chain fatty acids 2 (ELOVL2) and ELOVL5, which encode elongases 2 and 5 , respectively ${ }^{(40)}$. Furthermore, progesterone induces reduction in the DNA methylation of specific loci in the 5-regulatory region of its gene FADS2, which suggests that the difference in DHA synthesis and status between men and women may involve differential epigenetic regulation ${ }^{(40)}$. The biological significance of EPA and DHA synthesis in women has yet to be demonstrated directly.

\section{PUFA metabolism in women}

DHA status in women and rodents has been shown to increase during pregnancy ${ }^{(41-43)}$ and there is selective DHA enrichment in maternal hepatic and/or plasma phospholipids ${ }^{(43-46)}$ by a mechanism that involves altered acyl remodelling of phosphatidylcholine synthesised de novo ${ }^{(45)}$. Furthermore, the concentration of DHA in umbilical cord blood has been shown to be greater than that in the maternal circulation in humans ${ }^{(44,47)}$ and guinea pigs ${ }^{(48)}$, although the mechanism underlying the placental biomagnification of PUFA has not been characterised. Together, these processes may facilitate the supply of DHA from mother to offspring and buffer variation in supply of pre-formed DHA from the mother.

\section{EPA and DHA intakes in vegetarian and vegan women}

A number of governments and other organisations have published recommendations for combined intakes of EPA and DHA which range between $250^{(49)}$ and $1000 \mathrm{mg} / \mathrm{d}^{(50)}$, primarily to promote cardiovascular health. Relatively few of the studies that investigated the effect of vegetarian diets on EPA and DHA status in women have also reported the dietary intakes of these fatty acids. Welch $e t a l^{(51)}$ reported that UK vegetarian women consumed approximately $10 \mathrm{mg} / \mathrm{d}$ EPA and $<1 \mathrm{mg} / \mathrm{d}$ DHA, and that vegan women consumed 20 $\mathrm{mg} / \mathrm{d}$ EPA and no detectable DHA compared with fish-eating omnivore women (EPA $110 \mathrm{mg} / \mathrm{d}$; DHA $150 \mathrm{mg} / \mathrm{d}$ ). Lakin et al. ${ }^{(52)}$ estimated EPA and DHA intake of $17 \mathrm{mg} / \mathrm{d}$ in Scottish vegetarian mothers $(n 4)$ who had recently given birth compared with omnivore mothers $(316 \mathrm{mg} / \mathrm{d})$. These findings suggest that vegetarian and vegan women do not meet recommended EPA and DHA intakes and that their consumption of these fatty acids is markedly below that of their omnivore counterparts.

Only one study has reported the dietary sources of EPA and DHA in vegetarians and vegans ${ }^{(51)}$. Fat spreads accounted for $59 \%$ of EPA consumed by UK vegetarian women while a further $26 \%$ was provided by dairy foods and a further $6 \%$ by eggs and $6 \%$ from cereals. DHA was obtained from eggs $(88 \%)$ and from soups and sauces $(12 \%)$. In vegan women, EPA was obtained primarily from soups and sauces $(76 \%)$ and from spreading fats $(23 \%)$. DHA intake was too low to be measured in vegan women. Eggs and butter contain EPA and DHA. However, it is somewhat surprising that spreads and soups consumed by vegans, who do not consume dairy products or eggs, contained EPA since it is found only in foods derived from meat or oily fish. Unfortunately, the authors did not disclose which foods provided EPA in the diets of the vegan women.

ALNA intake in UK vegetarian women of $0.97 \mathrm{~g} / \mathrm{d}$ was similar to fish-eating omnivores $(0.99 \mathrm{~g} / \mathrm{d})$, although ALNA consumption in vegans $(0.86 \mathrm{~g} / \mathrm{d})$ was lower than both of these groups ${ }^{(51)}$. ALNA intake in Scottish vegetarian women $(1.5 \mathrm{~g} / \mathrm{d})$ was also similar to that of omnivores $(1.2 \mathrm{~g} / \mathrm{d})^{(52)}$. Linoleic acid intake was also similar between vegetarian (9.6 $\mathrm{g} / \mathrm{d}$ ) and omnivore women and the $18: 2 n-6 /$ ALNA ratio did not differ significantly between vegetarians $(7.0 \mathrm{~g} / \mathrm{d})$ and omnivores $(7.9 \mathrm{~g} / \mathrm{d})^{(52)}$. Although the evidence is limited, these findings suggest that, perhaps counter-intuitively, dietary intake of plant-derived essential fatty acids was not greater in vegetarians. This has implications for the influence of vegetarian diets on the capacity of vegetarian women to convert ALNA to longer-chain $n$-3 PUFA compared with omnivores. Since their intake of ALNA substrate and the relative intakes of essential fatty acids which compete for $\Delta 6$ desaturase were similar between the dietary groups, the capacity for synthesis of longer-chain $n-3$ PUFA may be expected to be comparable, except omnivores would be expected to have lower capacity for conversion of ALNA due to product inhibition by pre-formed dietary EPA and DHA.

Mead acid (20:3n-9) and DPAn-6 are markers of low essential fatty acid and DHA intakes, respectively ${ }^{(8,53)}$. The concentration of $20: 3 n-9$ did not differ significantly between ovo-lacto-vegetarians and omnivores, although only one study to date has reported the level of this fatty acid ${ }^{(54)}$. However, the concentration of $22: 5 n-6$ has been shown to be increased in ovo-lacto-vegetarians or vegans in one study ${ }^{(55)}$, but not others ${ }^{(52,56)}$. This suggests that at least some groups of vegetarians are at risk of DHA deficiency, although it is unclear why this cohort ${ }^{(55)}$ had an elevated $22: 5 n-6$ concentration compared with participants in other studies.

\section{EPA and DHA status in vegetarian and vegan women}

The findings of studies that compared EPA and DHA status in vegetarian women with individuals with other dietary practices are summarised in Table 1. These studies have been carried out in geographically diverse locations, with differing classifications of diet, and using different lipids (plasma or serum phospholipids, total serum fatty acids or erythrocyte membrane phospholipids) as the primary outcomes. Nevertheless, despite these potential confounding influences, consistent significant differences between vegetarians, vegans and those following other dietary practices have been reported.

Li et al. ${ }^{(57)}$ compared Australian vegetarian women (subjects who did not consume red meat and ate fish or chicken less than once per week for at least 6 months) and omnivores (defined as subjects who ate red meat at least five times per week); both groups aged 20 to 45 years. There were no significant differences in the proportions of ALNA or DPA $n-3$ in 
Table 1. Summary of studies that have compared $n-3$ PUFA status in omnivore and vegetarian (Mean values and standard deviations)

\begin{tabular}{|c|c|c|c|c|c|c|c|c|c|c|c|}
\hline \multirow[b]{2}{*}{ Study } & \multirow[b]{2}{*}{ Marker } & \multirow[b]{2}{*}{ Subjects and nationality } & \multirow[b]{2}{*}{$n$} & \multicolumn{2}{|c|}{ ALNA } & \multicolumn{2}{|c|}{ EPA } & \multicolumn{2}{|c|}{ DPA $n-3$} & \multicolumn{2}{|c|}{ DHA } \\
\hline & & & & Mean & SD & Mean & SD & Mean & SD & Mean & SD \\
\hline \multirow[t]{4}{*}{ Li et al. ${ }^{(57)}$} & $\begin{array}{l}\text { Serum phospholipids } \\
\text { (\% total fatty acids) }\end{array}$ & Australian & & & & & & & & & \\
\hline & & Omnivores & 24 & 0.23 & 0.10 & 0.95 & 0.46 & 0.85 & 0.22 & 3.51 & 0.64 \\
\hline & & Vegetarian & 50 & 0.24 & 0.10 & 0.60 & 0.29 & 0.91 & 0.25 & $2 \cdot 80$ & 0.86 \\
\hline & & Difference between groups $(P)$ & & \multicolumn{2}{|c|}{ NS } & \multicolumn{2}{|c|}{0.003} & \multicolumn{2}{|c|}{ NS } & \multicolumn{2}{|c|}{$<0.0001$} \\
\hline \multirow[t]{6}{*}{$\begin{array}{l}\text { Welch } \\
\quad \text { et al. }{ }^{(51)}\end{array}$} & $\begin{array}{l}\text { Plasma } \\
\text { phospholipids } \\
(\mu \mathrm{mol} / \mathrm{l})\end{array}$ & UK & & & & & & & & & \\
\hline & & Fish eaters & 1891 & $12 \cdot 4$ & $6 \cdot 1$ & 64.7 & 43.4 & 71.8 & 29.6 & 271.2 & $113 \cdot 1$ \\
\hline & & Meat eaters & 309 & $13 \cdot 1$ & $7 \cdot 3$ & $57 \cdot 1$ & $38 \cdot 4$ & $74 \cdot 7$ & $34 \cdot 2$ & 241.3 & $109 \cdot 6$ \\
\hline & & Vegetarians & 51 & $12 \cdot 3$ & $4 \cdot 8$ & $55 \cdot 1$ & $52 \cdot 5$ & $75 \cdot 0$ & $32 \cdot 2$ & 223.5 & $137 \cdot 8$ \\
\hline & & Vegans & 5 & 13.7 & $8 \cdot 1$ & $50 \cdot 0$ & $29 \cdot 4$ & $90 \cdot 6$ & $54 \cdot 0$ & $286 \cdot 4$ & $211 \cdot 7$ \\
\hline & & $\begin{array}{l}\text { Difference between groups } \\
\text { (ANOVA P) }\end{array}$ & & \multicolumn{2}{|c|}{ NS } & \multicolumn{2}{|c|}{$<0.001$} & \multicolumn{2}{|c|}{ NS } & \multicolumn{2}{|c|}{$<0.001$} \\
\hline \multirow[t]{4}{*}{$\begin{array}{l}\text { Melchert } \\
\text { et al. }{ }^{(58)}\end{array}$} & $\begin{array}{l}\text { Total serum lipids } \\
\text { (\% total serum } \\
\text { fatty acids) }\end{array}$ & Germany & & & & & & & & & \\
\hline & & Female non-vegetarians & 37 & $1 \cdot 10$ & 0.35 & \multicolumn{2}{|l|}{ ND } & \multicolumn{2}{|l|}{ ND } & $2 \cdot 36$ & 0.59 \\
\hline & & Female vegetarians & 38 & 1.35 & 0.81 & ND & & ND & & 1.42 & 0.47 \\
\hline & & Difference between groups $(P)$ & & \multicolumn{2}{|c|}{$<0.01$} & & & & & \multicolumn{2}{|c|}{$<0.001$} \\
\hline \multirow[t]{4}{*}{$\begin{array}{l}\text { Reddy } \\
\quad \text { et al. }{ }^{(59)}\end{array}$} & $\begin{array}{l}\text { Plasma } \\
\text { phospholipids } \\
\text { (\% total fatty acids) }\end{array}$ & UK & & & & & & & & & \\
\hline & & Caucasian omnivore & 24 & \multicolumn{2}{|l|}{ ND } & \multicolumn{2}{|l|}{0.97} & \multirow{2}{*}{\multicolumn{2}{|c|}{$\begin{array}{l}\text { ND } \\
\text { ND }\end{array}$}} & \multicolumn{2}{|l|}{$2 \cdot 26$} \\
\hline & & South Asian & 24 & ND & & 0.36 & & & & \multirow{2}{*}{\multicolumn{2}{|c|}{$\begin{array}{l}1.20 \\
<0.001\end{array}$}} \\
\hline & & Difference between groups $(P)$ & & & & \multicolumn{2}{|c|}{$<0.001$} & & & & \\
\hline
\end{tabular}

ALNA, $\alpha$-linolenic acid; ND, not determined.

* Mean values.

plasma phospholipids between vegetarians and omnivores (Table 1). However, there were lower proportions of EPA $(37 \%)$ and DHA (20\%) in plasma phospholipids in vegetarians compared with omnivores. Melchert et al. ${ }^{(58)}$ found that, in German women, the proportion of ALNA in total serum fatty acids was $23 \%$ higher and the proportion of DHA was $40 \%$ lower in vegetarians than in omnivores (Table 1). The proportions of EPA and DPA $n-3$ were not reported. Moreover, Reddy et al. ${ }^{(59)}$ showed that for South Asian vegetarian women living in the UK, EPA and DHA were not detected in their diets and that the proportion of EPA in plasma phospholipids was $63 \%$ and DHA $47 \%$ lower than in Caucasian omnivorous women (Table 1). The mean proportions of $n-3$ PUFA in plasma phospholipids across studies that used comparable measurements of fatty acid status ${ }^{(57-59)}$ were, for omnivores, ALNA $0.67 \%$, EPA $0.96 \%$, DPA $n-30.85 \%$ and DHA $3.0 \%$, and, for vegetarians, ALNA 0.80\%, EPA $0.48 \%$, DPA $n-3 \quad 0.90 \%$ and DHA $1.8 \%$ (Table 1). Vegetarians and vegans consumed less EPA and DHA, and had lower concentrations of these fatty acids in blood lipids than omnivores. However, in marked contrast to other studies, Welch et al. ${ }^{(51)}$ showed that despite a $25 \%$ lower intake of $\mathrm{EPA}+\mathrm{DPA} n-3+\mathrm{DHA}$ compared with fish-eating omnivores, vegan women in a cohort in the UK had $6 \%$ more $\mathrm{EPA}+\mathrm{DPA} n-3+\mathrm{DHA}$ in their plasma phospholipids than omnivore women (Table 1). One interpretation is that desaturation and elongation of ALNA to DHA was an important source of DHA in vegetarian and vegan women, although EPA and DHA biosynthesis appears to be insufficient to compensate completely for low intakes of these fatty acids. Furthermore, it cannot be concluded from these findings that low intakes of pre-formed EPA and DHA induce increased synthesis from ALNA and some authors have argued against the suggestion of up-regulation of EPA and DHA synthesis in vegetarians ${ }^{(60)}$.

\section{Effect of maternal vegetarian and vegan diets on EPA and DHA status in mothers and infants}

Adequate provision of DHA during early life is critical for development, particularly of the central nervous system ${ }^{(6)}$. Since DHA status and intakes are low among vegetarian and vegan women, it is possible that they may not be able to provide sufficient DHA to the fetus or infant to ensure adequate development.

To date, there have been few studies that have investigated specifically the effect of a maternal vegetarian or vegan diet on EPA and DHA status in infants. DHA concentration in umbilical cord plasma phospholipids was $32 \%$ lower in UK Hindu vegetarians ( $n$ 27) compared with matched Caucasian omnivores $^{(54)}$. These findings suggest that the supply of DHA from mother to fetus may be lower in vegetarians than omnivores. The same authors also showed that EPA and DHA intakes in lactating and non-lactating Hindu and Caucasian ovo-lacto-vegetarians were almost undetectable, and EPA and DHA intakes were too low to be measured in vegans. In contrast, omnivores consumed approximately 80 $\mathrm{mg}$ EPA/d and between 40 and $100 \mathrm{mg} \mathrm{DHA} / \mathrm{d}^{(54)}$. Others 
have also found EPA and DHA intakes to be below the level of detection in pregnant vegetarian women ${ }^{(61)}$.

The proportion of DHA in breast milk was $62 \%$ lower in vegans $(n 19)$ and $19 \%$ lower in Hindu vegetarians $(n 5)$ compared with omnivores $(n 21)^{(54)}$, presumably reflecting lower intake. Furthermore, the proportion of DHA in erythrocyte phospholipids in 14-week-old breast-fed infants of vegan mothers $(n 3)$ was $69 \%$ lower than that of omnivore mothers $(n 6)$, and was $49 \%$ lower than in infants who were fed cows' milk formula $(n 12)^{(54)}$, although it is difficult to be confident that this small number of subjects is representative of the wider vegetarian population. However, the same group also reported $80 \%$ lower EPA concentration in breast milk of Caucasian vegans compared with that of omnivores ${ }^{(55)}$. Mead acid represented $0 \cdot 2 \%$ total fatty acids in erythrocytes from infants breast-fed by omnivore women ( $n$ 3), but only a trace amount was detected in infants of omnivores $(n 6)^{(54)}$. This may reflect the lower concentrations of ALNA (47\%) and linoleic acid $(78 \%)$ in breast milk of omnivores compared with that of vegan women. Together, the findings of these studies suggest that despite women being able to convert ALNA to EPA and DHA, and metabolic adaptations that increase maternal DHA concentration during pregnancy, vegan and vegetarian women do not appear to able to compensate metabolically for low intakes of pre-formed EPA and DHA during pregnancy.

Lakin et al. ${ }^{(52)}$ found that in Scottish vegetarian mothers $(n 4)$ the proportion of EPA was $30 \%$ and of DHA $15 \%$ lower in total placental lipids compared with omnivore $(n 10)$ mothers. In addition, EPA was undetectable and DHA $43 \%$ lower in umbilical cord tissues from vegetarian pregnancies compared with omnivores ${ }^{(52)}$. Reddy et al. ${ }^{(59)}$ have shown that the proportions of EPA and DHA in erythrocyte phospholipids of 14-week-old infants breast-fed by vegan mothers $(n$ 3) were lower than in infants breast-fed by omnivore mothers (EPA $71 \%$; DHA $40 \%$ ) or born to omnivore mothers and fed cows' milk formula (EPA $83 \%$; DHA $49 \%$ ). These findings were confirmed by Sanders et al. ${ }^{(55)}$ who showed that the proportions of EPA and DHA in erythrocytes of infants of at least 3 months of age breast-fed by vegan mothers $(n 3)$ were lower (EPA $71 \%$; DHA $69 \%$ ) than those of infants breast-fed by omnivore mothers $(n$ 6). Together these data suggest that adaptions to maternal PUFA metabolism that increase DHA status during pregnancy ${ }^{(43)}$, the capacity of women to convert ALNA to longer-chain $n-3$ PUFA $^{(36)}$ and biomagnification of DHA by the placenta ${ }^{(44,47)}$ were not able to compensate for the absence of pre-formed EPA and DHA either before birth and during breast-feeding. Since low intakes of DHA during infancy have been associated with a deficit in the accumulation of DHA in brain phospholipids in term infants ${ }^{(62,63)}$, these findings suggest a potential concern about a negative impact of maternal vegetarian, in particular the vegan diet, on the development of the central nervous system of infants.

\section{Vegetarian diets, DHA and child development}

To date, there have not been any studies to determine whether low DHA status in infants of vegetarian or vegan mothers has any significant adverse effect on their development ${ }^{(64)}$ and it has been argued that lifelong DHA insufficiency in vegans may increase risk of cognitive decline ${ }^{(65)}$. However, some insights may be gained from studies of the effect of low maternal fish intake on infant development. One study has investigated the relationship between maternal fish intake and $n-3$ long-chain PUFA status in pregnant Indian women and the birth weight of their infants ${ }^{(66)}$. Women who did not consume fish during their third trimester had an adjusted OR of 2.49 $(95 \%$ CI $1 \cdot 16,5 \cdot 36 ; P=0.019)$ for the risk of delivering a low-birth-weight infant and low DHA during pregnancy has been implicated in an increased risk of preterm delivery ${ }^{(67)}$. Others have also shown that low consumption of seafood is a risk factor for preterm delivery ${ }^{(68)}$. In addition, low umbilical cord plasma DHA concentration has been associated with impaired mental and psychomotor development at the age of 6 months ${ }^{(69)}$. However, without appropriate assessment of neurological development in children of vegetarian mothers and other potential confounding factors such as socioeconomic status, it is not possible to conclude whether low DHA intake and status affect the neurological development of children negatively.

The potential risk of impaired development as a consequence of low DHA supply from mother to fetus is of particular importance to populations that consume predominately a vegetarian diet. For example, the diet of the Indian population is based mainly on cereals with some use of ALNA-rich oils ${ }^{(70)}$. The lack of pre-formed EPA and DHA in the diet may, therefore, have a negative impact on development and wellbeing at the population level, although at present there is no evidence to support or refute this suggestion. A recent study has examined allele frequency of the FADS2 rs66698963 polymorphism in 234 Indian vegetarians and 311 Americans ${ }^{(71)}$. This 22-bp insertion/deletion mutation has been associated with basal FADS1 expression, and with up-regulation of fatty acid desaturase genes FADS1 and FADS2 ${ }^{(72)}$. Kothapalli et al. ${ }^{(71)}$ found that the insertion allele, which was associated with increased expression of FADS1 and FADS2, was found in $68 \%$ Indians, compared with $18 \%$ of Americans. Thus, this allele seems to have evolved in populations that predominantly consumed a plant-based diet for millennia and may enable them to convert $n-3$ and $n-6$ fatty acids to long-chain PUFA more efficiently than in populations that habitually consume omnivorous diets (although the study only focused on $n-6$ PUFA).

\section{Dietary supplementation with $\mathrm{n}-3$ PUFA in vegetarians}

Dietary supplementation with EPA and DHA to improve status presents a challenge in non-fish-eating vegetarians and vegans. TAG extracted from marine algae represent one potential source of EPA and DHA that is consistent with vegetarian diets ${ }^{(73)}$. Unsurprisingly, consuming algal oil supplements increased the concentration of DHA in blood from vegetarian men and/or women ${ }^{(35,65,74-76)}$, although one study showed an increase in total and LDL-cholesterol concentration following the intervention ${ }^{(75)}$. One study has investigated the effect of a food-based dietary intervention on EPA and DHA status in North American lacto-ovo-vegetarian men 
and women ${ }^{(77)}$. Subjects consumed either DHA-enriched eggs (DHA $500 \mathrm{mg}$, EPA $40 \mathrm{mg}$, ALNA $1 \mathrm{~g} /$ yolk) or standard eggs (DHA $110 \mathrm{mg}$, EPA negligible, ALNA 0.15 g). The DHA-enriched eggs increased dietary DHA intake by 4.5-fold compared with the standard egg diets. Consumption of enriched eggs increased the proportion of DHA, but not EPA, in erythrocyte phospholipids. Another group in the same study consumed walnuts ( $2.95 \mathrm{~g}$ ALNA) which provided 3 -fold more ALNA than the standard egg diet. Consuming walnuts was associated with a significant decrease in the proportion of DHA in erythrocyte phospholipids, but no change in the proportion of EPA in contrast to other studies in which ALNA intake was increased ${ }^{(33)}$. These studies show that specific dietary strategies that are potentially acceptable by vegetarians or vegans are effective in increasing EPA and DHA status.

\section{Conclusions}

Studies published to date show, with few exceptions, that EPA and DHA intakes and status in vegetarians and vegans are lower than in omnivores. Synthesis of EPA and DHA may be an important source of these fatty acids in vegetarians and, in particular, vegan women and there is no evidence of metabolic compensation for low intakes of EPA and DHA. In addition, infants born to vegan mothers have lower EPA and DHA status than those born to omnivore mothers. However, the interpretation of these is constrained by the relatively few studies of pregnant and non-pregnant vegetarian women and their offspring. Furthermore, the studies of EPA and DHA status in infants have included very small numbers of subjects and so there is a risk of confounding and of limited extrapolation to the wider population. Furthermore, there have not been any studies of the effect of low DHA status due to maternal vegetarian or vegan diets on cognitive function in children. This represents an important knowledge gap which needs to be addressed in order to be confident that vegetarian and vegan diets during pregnancy are safe in the context of child development and to be able to make appropriate nutritional recommendations.

\section{Acknowledgements}

This review was not supported by a specific source of funding.

The authors' contributions are as follows: G. C. B. and C. J. H. conceived the idea and wrote the review, and S.-Y. T. co-authored the review.

G. C. B. is a member of the Scientific Advisory Board of BASF and a member of the Board of Directors of the International Society for the Study of Fatty Acids and Lipids (ISSFAL). He has received research funding from Nestlé, Danone and Abbott Nutrition.

\section{References}

1. Corrin T \& Papadopoulos A (2017) Understanding the attitudes and perceptions of vegetarian and plant-based diets to shape future health promotion programs. Appetite 109, 40-47.
2. Tur JA, Bibiloni MM, Sureda A, et al. (2012) Dietary sources of omega 3 fatty acids: public health risks and benefits. Br J Nutr 107, Suppl. 2, S23-S52.

3. Calder PC (2015) Marine omega-3 fatty acids and inflammatory processes: effects, mechanisms and clinical relevance. Biochim Biophy Acta 1851, 469-484.

4. Calder PC \& Yaqoob P (2009) Omega-3 polyunsaturated fatty acids and human health outcomes. Biofactors 35, 266-272.

5. Harris WS \& Von Schacky C (2004) The Omega-3 Index: a new risk factor for death from coronary heart disease? Prev Med 39, 212-220.

6. Lauritzen L, Brambilla P, Mazzocchi A, et al. (2016) DHA effects in brain development and function. Nutrients 8,6 .

7. Carlson SE \& Neuringer M (1999) Polyunsaturated fatty acid status and neurodevelopment: a summary and critical analysis of the literature. Lipids 34, 171-178.

8. Connor WE, Neuringer M \& Lin DS (1990) Dietary effects on brain fatty acid composition: the reversibility of $n-3$ fatty acid deficiency and turnover of docosahexaenoic acid in the brain, erythrocytes, and plasma of rhesus monkeys. J Lipid Res 31, 237-247.

9. Reisbick S, Neuringer M, Hasnain R, et al. (1994) Home cage behavior of rhesus monkeys with long-term deficiency of omega-3 fatty acids. Physiol Behav 55, 231-239.

10. Reisbick S, Neuringer M, Hasnain R, et al. (1990) Polydipsia in rhesus monkeys deficient in omega-3 fatty acids. Physiol Behav 47, 315-323.

11. Sanders TA (2009) DHA status of vegetarians. Prostaglandins Leukot Essent Fatty Acids 81, 137-141.

12. Sanders TA (1981) Vegetarian diets. Nurs Times 77, 446-447.

13. Sanders $\mathrm{T}$ (1994) Good nutrition for the vegetarian mother. Mod Midwife 4, 23-26.

14. Sanders TA (1995) Vegetarian diets and children. Pediatr Clin North Am 42, 955-965.

15. Sanders TA (2014) Plant compared with marine $n$-3 fatty acid effects on cardiovascular risk factors and outcomes: what is the verdict? Am J Clin Nutr 100, Suppl. 1, 453S-458S.

16. Wang F, Zheng J, Yang B, et al. (2015) Effects of vegetarian diets on blood lipids: a systematic review and meta-analysis of randomized controlled trials. J Am Heart Assoc 4, e002408.

17. Chiu TH, Huang HY, Chiu YF, et al. (2014) Taiwanese vegetarians and omnivores: dietary composition, prevalence of diabetes and IFG. PLOS ONE 9, e88547.

18. Tonstad S, Stewart K, Oda K, et al. (2013) Vegetarian diets and incidence of diabetes in the Adventist Health Study-2. Nutr Metab Cardiovasc Dis 23, 292-299.

19. Sabate J \& Wien M (2015) A perspective on vegetarian dietary patterns and risk of metabolic syndrome. Br J Nutr 113, Suppl. 2, S136-S143.

20. Huang RY, Huang CC, Hu FB, et al. (2016) Vegetarian diets and weight reduction: a meta-analysis of randomized controlled trials. J Gen Intern Med 31, 109-116.

21. Orlich MJ, Singh PN, Sabate J, et al. (2015) Vegetarian dietary patterns and the risk of colorectal cancers. JAMA Intern Med 175, 767776.

22. Dinu M, Abbate R, Gensini GF, et al. (2016) Vegetarian, vegan diets and multiple health outcomes: a systematic review with meta-analysis of observational studies. Crit Rev Food Sci Nutr 57, 3640-3649.

23. Glick-Bauer M \& Yeh MC (2014) The health advantage of a vegan diet: exploring the gut microbiota connection. Nutrients 6, 48224838.

24. Piccoli GB, Clari R, Vigotti FN, et al. (2015) Vegan-vegetarian diets in pregnancy: danger or panacea? A systematic narrative review. BJOG 122, 623-633.

25. Lohner S, Fekete K, Marosvolgyi T, et al. (2013) Gender differences in the long-chain polyunsaturated fatty acid status: systematic review of 51 publications. Ann Nutr Metab 62, 98-112.

26. Burdge $G$ (2004) $\alpha$-Linolenic acid metabolism in men and women: nutritional and biological implications. Curr Opin Clin Nutr Metab Care 7, 137-144. 
27. Burdge GC, Jones AE \& Wootton SA (2002) Eicosapentaenoic and docosapentaenoic acids are the principal products of $\alpha$-linolenic acid metabolism in young men. Br J Nutr 88, 355-363.

28. Murphy DJ \& Stumpf PK (1979) Elongation pathway for $\alpha$-linolenic acid synthesis in spinach leaves: a reexamination. Plant Physiol 64, 428-430.

29. Griffiths G, Brechany EY, Jackson FM, et al. (1996) Distribution and biosynthesis of stearidonic acid in leaves of Borago officinalis. Phytochemistry 43, 381-386.

30. Baker EJ, Miles EA, Burdge GC, et al. (2016) Metabolism and functional effects of plant-derived omega-3 fatty acids in humans. Prog Lipid Res 64, 30-56.

31. Yamanaka WK, Clemans GW \& Hutchinson ML (1980) Essential fatty acids deficiency in humans. Prog Lipid Res 19, 187-215.

32. Voss AC \& Sprecher H (1988) Regulation of the metabolism of linoleic acid to arachidonic acid in rat hepatocytes. Lipids 23, 660-665.

33. Burdge GC \& Calder PC (2006) Dietary $\alpha$-linolenic acid and health-related outcomes: a metabolic perspective. Nutr Res Rev 19, 26-52.

34. James MJ, Ursin VM \& Cleland LG (2003) Metabolism of stearidonic acid in human subjects: comparison with the metabolism of other $n$-3 fatty acids. Am J Clin Nutr 77, 1140-1145.

35. Conquer JA \& Holub BJ (1997) Dietary docosahexaenoic acid as a source of eicosapentaenoic acid in vegetarians and omnivores. Lipids 32, 341-345.

36. Burdge GC \& Wootton SA (2002) Conversion of $\alpha$-linolenic acid to eicosapentaenoic, docosapentaenoic and docosahexaenoic acids in young women. Br J Nutr 88, 411-420.

37. Toledo A, Andersson MN, Wang HL, et al. (2016) Fatty acid profiles of great tit (Parus major) eggs differ between urban and rural habitats, but not between coniferous and deciduous forests. Naturwissenschaften 103, 55.

38. Giltay EJ, Gooren LJ, Toorians AW, et al. (2004) Docosahexaenoic acid concentrations are higher in women than in men because of estrogenic effects. Am J Clin Nutr 80, 1167-1174.

39. Giltay EJ, Duschek EJ, Katan MB, et al. (2004) Raloxifene and hormone replacement therapy increase arachidonic acid and docosahexaenoic acid levels in postmenopausal women. J Endocrinol 182, 399-408.

40. Sibbons CM, Brenna JT, Lawrence P, et al. (2014) Effect of sex hormones on $n-3$ polyunsaturated fatty acid biosynthesis in HepG2 cells and in human primary hepatocytes. Prostaglandins Leukot Essent Fatty Acids 90, 47-54.

41. Childs CE, Hoile SP, Burdge GC, et al. (2012) Changes in rat $n-3$ and $n-6$ fatty acid composition during pregnancy are associated with progesterone concentrations and hepatic FADS2 expression. Prostaglandins Leukot Essent Fatty Acids 86, 141-147.

42. Childs CE, Romeu-Nadal M, Burdge GC, et al. (2010) The polyunsaturated fatty acid composition of hepatic and plasma lipids differ by both sex and dietary fat intake in rats. J Nutr 140, 245-250.

43. Postle AD, Al MD, Burdge GC, et al. (1995) The composition of individual molecular species of plasma phosphatidylcholine in human pregnancy. Early Hum Dev 43, 47-58.

44. Burdge GC, Sherman RC, Ali Z, et al. (2006) Docosahexaenoic acid is selectively enriched in plasma phospholipids during pregnancy in Trinidadian women - results of a pilot study. Reprod Nutr Dev 46, 63-67.

45. Burdge GC, Hunt AN \& Postle AD (1994) Mechanisms of hepatic phosphatidylcholine synthesis in adult rat: effects of pregnancy. Biochem J 303, 941-947.

46. Burdge GC \& Postle AD (1994) Hepatic phospholipid molecular species in the guinea pig. Adaptations to pregnancy. Lipids 29, 259-264.

47. Crawford MA, Golfetto I, Ghebremeskel K, et al. (2003) The potential role for arachidonic and docosahexaenoic acids in protection against some central nervous system injuries in preterm infants. Lipids 38, 303-315.

48. Crawford MA, Hassam AG \& Williams G (1976) Essential fatty acids and fetal brain growth. Lancet $\mathbf{i}, 452-453$.
49. EFSA Panel on Dietetic Products, Nutrition, and Allergies (NDA) (2010) Scientific opinion on dietary reference values for fats, including saturated fatty acids, polyunsaturated fatty acids, monounsaturated fatty acids, trans fatty acids, and cholesterol. EFS A J 8, 1461.

50. Japanese Ministry of Health, Labour and Welfare (2009) Dietary Reference Intakes for Japanese, 2010 revision. Summary report. http://www.nibiohn.go.jp/en/files/Section_of_the_Dietary_Reference_ Intakes/dris2010_eng.pdf

51. Welch AA, Shakya-Shrestha S, Lentjes MA, et al. (2010) Dietary intake and status of $n-3$ polyunsaturated fatty acids in a population of fisheating and non-fish-eating meat-eaters, vegetarians, and vegans and the precursor-product ratio of $\alpha$-linolenic acid to long-chain $n$ - 3 polyunsaturated fatty acids: results from the EPIC-Norfolk cohort. $A m \mathrm{~J}$ Clin Nutr 92, 1040-1051.

52. Lakin V, Haggarty P, Abramovich DR, et al. (1998) Dietary intake and tissue concentration of fatty acids in omnivore, vegetarian and diabetic pregnancy. Prostaglandins Leukot Essent Fatty Acids 59, 209 220.

53. Mead JF \& Slaton WH Jr (1956) Metabolism of essential fatty acids. III. Isolation of 5,8,11-eicosatrienoic acid from fat-deficient rats. J Biol Chem 219, 705-709.

54. Sanders TA \& Reddy S (1992) The influence of a vegetarian diet on the fatty acid composition of human milk and the essential fatty acid status of the infant. J Pediatr 120, S71-S77.

55. Sanders TA, Ellis FR \& Dickerson JW (1978) Studies of vegans: the fatty acid composition of plasma choline phosphoglycerides, erythrocytes, adipose tissue, and breast milk, and some indicators of susceptibility to ischemic heart disease in vegans and omnivore controls. Am J Clin Nutr 31, 805-813.

56. Huang T, Yu X, Shou T, et al. (2013) Associations of plasma phospholipid fatty acids with plasma homocysteine in Chinese vegetarians. Br J Nutr 109, 1688-1694.

57. Li D, Ball M, Bartlett M, et al. (1999) Lipoprotein(a), essential fatty acid status and lipoprotein lipids in female Australian vegetarians. Clin Sci (Lond) 97, 175-181.

58. Melchert HU, Limsathayourat N, Mihajlovic H, et al. (1987) Fatty acid patterns in triglycerides, diglycerides, free fatty acids, cholesteryl esters and phosphatidylcholine in serum from vegetarians and non-vegetarians. Atherosclerosis 65, 159-166.

59. Reddy S, Sanders TA \& Obeid O (1994) The influence of maternal vegetarian diet on essential fatty acid status of the newborn. Eur J Clin Nutr 48, 358-368.

60. Salem N Jr \& Kuratko CN (2011) Lack of evidence for increased $\alpha$-linolenic acid metabolism in vegetarians. Am J Clin Nutr 93, 1154-1155; author reply 1155-1156.

61. Stammers JP, Hull D, Abraham R, et al. (1989) High arachidonic acid levels in the cord blood of infants of mothers on vegetarian diets. Br J Nutr 61, 89-97.

62. Farquharson J, Jamieson EC, Abbasi KA, et al. (1995) Effect of diet on the fatty acid composition of the major phospholipids of infant cerebral cortex. Arch Dis Child 72, 198-203.

63. Martinez M (1992) Tissue levels of polyunsaturated fatty acids during early human development. J Pediatr 120, S129-S138.

64. Stonehouse W (2014) Does consumption of LC omega-3 PUFA enhance cognitive performance in healthy school-aged children and throughout adulthood? Evidence from clinical trials. Nutrients 6, 2730-2758.

65. Sarter B, Kelsey KS, Schwartz TA, et al. (2015) Blood docosahexaenoic acid and eicosapentaenoic acid in vegans: associations with age and gender and effects of an algal-derived omega- 3 fatty acid supplement. Clin Nutr 34, 212-218.

66. Muthayya S, Dwarkanath P, Thomas T, et al. (2009) The effect of fish and omega-3 LCPUFA intake on low birth weight in Indian pregnant women. Eur J Clin Nutr 63, 340-346.

67. Kar S, Wong M, Rogozinska E, et al. (2016) Effects of omega-3 fatty acids in prevention of early preterm delivery: a systematic review and meta-analysis of randomized studies. Eur J Obstet Gynecol Reprod Biol 198, 40-46. 
68. Olsen SF \& Secher NJ (2002) Low consumption of seafood in early pregnancy as a risk factor for preterm delivery: prospective cohort study. BMJ 324, 447.

69. Zornoza-Moreno M, Fuentes-Hernández S, Carrión V, et al. (2014) Is low docosahexaenoic acid associated with disturbed rhythms and neurodevelopment in offsprings of diabetic mothers? Eur J Clin Nutr 68, 931-937.

70. Rastogi T, Reddy KS, Vaz M, et al. (2004) Diet and risk of ischemic heart disease in India. Am J Clin Nutr 79, 582-592.

71. Kothapalli KS, Ye K, Gadgil MS, et al. (2016) Positive selection on a regulatory insertion-deletion polymorphism in FADS2 influences apparent endogenous synthesis of arachidonic acid. Mol Biol Evol 33, 1726-1739.

72. Reardon HT, Zhang J, Kothapalli KS, et al. (2012) Insertion-deletions in a FADS2 intron 1 conserved regulatory locus control expression of fatty acid desaturases 1 and 2 and modulate response to simvastatin. Prostaglandins Leukot Essent Fatty Acids 87, 25-33.
73. Salem N Jr \& Eggersdorfer M (2015) Is the world supply of omega-3 fatty acids adequate for optimal human nutrition? Curr Opin Clin Nutr Metabol Care 18, 147-154.

74. Geppert J, Kraft V, Demmelmair H, et al. (2005) Docosahexaenoic acid supplementation in vegetarians effectively increases omega-3 index: a randomized trial. Lipids 40, 807-814.

75. Geppert J, Kraft V, Demmelmair H, et al. (2006) Microalgal docosahexaenoic acid decreases plasma triacylglycerol in normolipidaemic vegetarians: a randomised trial. Br J Nutr 95, 779-786.

76. Wu WH, Lu SC, Wang TF, et al. (2006) Effects of docosahexaenoic acid supplementation on blood lipids, estrogen metabolism, and in vivo oxidative stress in postmenopausal vegetarian women. Eur J Clin Nutr 60, 386-392.

77. Burns-Whitmore B, Haddad E, Sabate J, et al. (2014) Effects of supplementing $n$-3 fatty acid enriched eggs and walnuts on cardiovascular disease risk markers in healthy free-living lacto-ovo-vegetarians: a randomized, crossover, free-living intervention study. Nutr J13, 29. 